PAPER

\title{
Neuropsychological effects associated with temporal lobectomy and amygdalohippocampectomy depending on Wada test failure
}

\author{
M E Lacruz, G Alarcón, N Akanuma, F C K Lum, N Kissani, M Koutroumanidis, N Adachi, \\ C D Binnie, C E Polkey, R G Morris
}

J Neurol Neurosurg Psychiatry 2004;75:600-607. doi: 10.1136/jnnp.2003.020248

See end of article for authors' affiliations

.....................

Correspondence to:

Dr G Alarcón, Department of Clinical

Neurophysiology, King's

College Hospital, 'Denmark Hill, London SE5 9RS, UK; galarcon@aol.com

Received 7 June 2003

In revised form

8 September 2003

Accepted

11 September 2003

\begin{abstract}
Objective: To compare the neuropsychological effects of temporal lobectomy (TL) and amygdalohippocampectomy (AH), depending on whether the patients had passed or failed the Wada test.

Methods: We compared changes in neuropsychological scores in patients who underwent TL $(n=91)$ or $\mathrm{AH}(\mathrm{n}=15)$, and had passed or failed the Wada test. Comparisons were carried out in all 106 patients and among the 20 patients who failed the Wada test ( 12 who had TL and 8 who had AH).

Results: No patient became globally amnesic after surgery. Among all patients, no differences were found in pre-surgical or change scores (percentage of change after surgery compared with preoperative values) of neuropsychological tests between patients who underwent TL or AH. Among patients who failed the Wada test, those in the TL group showed higher visual memory impairment $(p<0.05)$. There was a strong trend suggesting that $T L$ is associated with higher verbal memory deficits than $A H(p=0.07)$. Of those TL patients who failed the Wada test, the contralateral Wada score correlated with change scores in verbal intelligence quotient $(p<0.01)$, and there was a strong trend towards a correlation with the logical memory immediate recall version subtest of the Wechsler Memory Scale $(p=0.06)$.

Conclusions: No profound changes in intelligence quotient or memory scores were found after TL or AH. Nevertheless, patients who underwent TL and failed the Wada test showed more deficits than those who passed the test or those who had $\mathrm{AH}$. The presence of a correlation between contralateral Wada scores and verbal deficits in TL patients who failed the Wada test but not among AH patients suggests that, if temporal surgery is required, $\mathrm{AH}$ might be preferred to $\mathrm{TL}$ in patients who fail the Wada test.
\end{abstract}

A lthough temporal lobe resective surgery has long been used effectively for the treatment of temporal lobe epilepsy refractory to medication, the neuropsychological effects of temporal resections are still debated. After Scoville and Milner reported on a patient who developed severe anterograde amnesia following bilateral medial temporal lobectomy, ${ }^{1}$ it became widely accepted that medial temporal structures mediate declarative memory in humans. $^{23}$

Nevertheless, the effects of unilateral temporal resections on human memory remain controversial. Resective surgery has long been used for the treatment of temporal lobe epilepsy refractory to medication. Since the initial description at our centre, ${ }^{4}$ en bloc temporal lobectomy (TL) has become one of the standard procedures for the surgical treatment of temporal lobe epilepsy. A more restricted resection, the amygdalohippocampectomy $(\mathrm{AH})$ has been proposed as an alternative to TL in patients where seizures arise from mesial temporal structures, particularly within the hemisphere that mainly supports memory, as it is thought that $\mathrm{AH}$ induces fewer cognitive deficits than TL. ${ }^{5-7}$

Few rigorous studies have compared the cognitive sequelae of TL and AH. In 1982, Wieser et al compared five patients who underwent unilateral removal of the anterior two thirds of the temporal lobe with six patients who had AH. ${ }^{5}$ They showed that verbal memory deficits followed left TL but not left $\mathrm{AH}$, and that right $\mathrm{TL}$ was associated with more pronounced impairment in visuospatial learning than right AH. Goldstein et al compared patients who underwent TL or $\mathrm{AH}$ in two studies. ${ }^{89}$ In the first, with 46 patients, verbal and visuospatial memory and learning were less impaired after
AH than after TL. In the second, with 42 patients, there was no evidence that TL produced more pronounced everyday memory impairment than $\mathrm{AH}^{8}{ }^{8}$ Helmstaedter et al found significant deterioration on several aspects of long term verbal memory after both procedures if carried out in the dominant hemisphere. ${ }^{10}{ }^{11}$ In addition, patients who underwent TL exhibited deterioration in short term verbal memory. Pauli et al, comparing verbal and non-verbal scores of the Wechsler Memory Scale Revised from 69 patients who underwent temporal resections of different sizes, reported significant verbal memory declines after TL but not after AH. ${ }^{12}$ In contrast, Wolf et al found no differences in neuropsychological performance depending on the extent of mesial and lateral left temporal resections, suggesting that the extent of left temporal resections has minimal impact on cognitive outcome. ${ }^{13}$ Finally, Jones-Gotman et al found similar deficits after unilateral neocorticectomy, $\mathrm{AH}$, or TL on verbal and non-verbal learning tasks. ${ }^{14}$

In principle, differences in cognitive function after each type of temporal resection could be due to differences in seizure control after surgery. Nevertheless, there appears to be no difference in post-surgical seizure control between patients who undergo TL and patients showing mesial temporal seizure onset who undergo AH. ${ }^{75}{ }^{16}$ Consequently,

Abbreviations: $\mathrm{AH}$, amygdalohippocampectomy; $\mathrm{FSIQ}$, full scale intelligence quotient; $P I Q$, performance intelligence quotient; TL, temporal lobectomy; VIQ, verbal intelligence quotient; WLMD, Wechsler logical memory (delayed); WLMI, Wechsler logical memory

(immediate); WMS, Wechsler Memory Scale 
Table 1 Demographic characteristics of TL and AH groups (all patients and those who failed the Wada test)

\begin{tabular}{|c|c|c|c|c|c|c|}
\hline & \multicolumn{3}{|l|}{ All patients } & \multicolumn{3}{|l|}{ Failed Wada test } \\
\hline & TL & $\mathrm{AH}$ & Total & TL & $\mathbf{A H}$ & Total \\
\hline Number of patients & 91 & 15 & 106 & 12 & 8 & 20 \\
\hline Operation side (left/right) & $45 / 46$ & $9 / 6$ & $54 / 52$ & $7 / 5$ & $5 / 3$ & $12 / 8$ \\
\hline $\operatorname{Sex}(M / F)$ & $50 / 41$ & $9 / 6$ & $59 / 47$ & $4 / 8$ & $6 / 2$ & $10 / 10$ \\
\hline Handedness (right/non-right) & $78 / 13$ & $12 / 3$ & $90 / 16$ & $9 / 3$ & $7 / 1$ & $16 / 4$ \\
\hline \multicolumn{7}{|c|}{ Wada test } \\
\hline $\begin{array}{l}\text { Memory laterality (unilateral low/ } \\
\text { bilateral high/bilateral low) }\end{array}$ & $41 / 38 / 12^{*}$ & $2 / 5 / 8^{*}$ & $48 / 43 / 15$ & $2 / 0 / 10$ & $3 / 0 / 5$ & $5 / 0 / 15$ \\
\hline $\begin{array}{l}\text { Language dominance (left/right/ } \\
\text { bilateral/not established) }\end{array}$ & $73 / 5 / 11 / 2$ & $14 / 1 / 0 / 0$ & $87 / 6 / 11 / 2$ & $10 / 1 / 1$ & $8 / 0 / 0$ & $18 / 1 / 1$ \\
\hline Passed/failed & $79 / 12 \dagger$ & $7 / 8 \dagger$ & $86 / 20$ & $0 / 12$ & $0 / 8$ & $0 / 20$ \\
\hline Histopathology (MTS/non-MTS) & $63 / 28 \ddagger$ & $13 / 2 \ddagger$ & $76 / 30$ & $8 / 4$ & $7 / 1$ & $15 / 5$ \\
\hline $\begin{array}{l}\text { Post-surgical seizure control } \\
\text { (favourable/poor) }\end{array}$ & $58 / 33$ & $9 / 6$ & $67 / 39$ & $7 / 5$ & $5 / 3$ & $12 / 8$ \\
\hline Age at onset of epilepsy (years) & $7(0.5$ to 45$)$ & $6(1$ to 25$)$ & $6.5(0.5$ to 45$)$ & $5.5(0.5$ to 19$)$ & 7.2 (1 to 25$)$ & $5.5(0.5$ to 25$)$ \\
\hline Age at operation (years) & $28(13$ to 58$)$ & $26(20$ to 44$)$ & 27.5 (13 to 58$)$ & 27.5 (19 to 49$)$ & 28 (23 to 39$)$ & 27.5 (19 to 49$)$ \\
\hline Duration of epilepsy (years) & $18(2$ to 46$)$ & 21 (7 to 42$)$ & $18.5(2$ to 46$)$ & $17.5(12$ to 40$)$ & 21.2 (12 to 28$)$ & $19.5(12$ to 40$)$ \\
\hline Contralateral Wada scores & $9(-6$ to 17$) \S$ & $4(-1$ to 10$) \S$ & $8.5(-6$ to 17$)$ & $2(-6$ to 4$)$ & $1.5(-1$ to 4$)$ & $2(-6$ to 4$)$ \\
\hline Ipsilateral Wada scores & $4(-3$ to 14$)$ & $5(0$ to 10$)$ & $4(-3$ to 14$)$ & $0.5(-3$ to 7$)$ & $3(0$ to 10$)$ & $1(-3$ to 10$)$ \\
\hline
\end{tabular}

the main consideration in deciding to carry out either operation stems from the initial suggestion that $\mathrm{AH}$ might preserve memory in patients where the Wada test has suggested that memory is not supported by the hemisphere contralateral to the operation. This rationale is based on the assumption that TL removes tissue relevant to memory. The Wada tes $\mathrm{t}^{17-19}$ is used routinely in our centre to estimate and avoid potential memory and speech deficits associated with temporal lobe surgery. Unilateral intracarotid injection of sodium amobarbital prior to performance of memory tests allows estimation of unilateral memory and speech functions, permitting a prediction of the neuropsychological effects of a unilateral temporal resection. Thus, memory and speech functions observed after injecting the hemisphere ipsilateral to the proposed operation are regarded as the main predictors for post-operative memory and speech deficits. Lower memory Wada scores and more severe dysphasia after injecting the side of proposed surgery are believed to predict greater post-operative deficits after TL. In our centre, TL was the operation of first choice for the treatment of temporal lobe epilepsy and was performed in most patients. AH was considered for those patients with poor memory on the non-operated hemisphere because it was assumed to induce fewer cognitive deficits as it removes less cortex.

In the present work we have compared the neuropsychological effects of TL and $\mathrm{AH}$ in order to validate the following hypotheses:

- Patients who fail the Wada test are at greater risk of memory loss after a temporal resection;

- TL induces more memory deficits than AH.

As the basic difference between TL and AH is a different degree of neocortical removal, the comparison of neuropsychological deficits associated with each procedure could provide an estimate of the neocortical contribution to cognitive function.

We compared results from 91 patients who underwent TL and 15 who underwent $\mathrm{AH}$ at our centre between 1987 and 1999. As the inclusion criteria for AH are much stricter than for TL (see Methods), the number of patients undergoing AH was necessarily smaller than the number undergoing TL. In contrast to most previous reports, we were able to include TL and AH patients who failed the Wada test after injecting the hemisphere ipsilateral to the operation (that is, those patients with poor memory on the non-resected side). This comparison helped us to clarify the indications of AH for epilepsy surgery. We present neuropsychological findings from 12 patients who had a TL and 8 who had AH despite failing the Wada test on injection of the operated hemisphere. The 12 patients who had TL despite failing the Wada test when injecting the operated side are of particular relevance because these are the patients thought to be at greatest risk of developing memory deficits. However, it should be emphasised that in our study, these patients represent a very select population where we thought that TL was justified (see selection criteria for TL in Methods).

\section{PATIENTS AND METHODS Patients}

The following inclusion criteria were used: (a) patients underwent TL or AH for the treatment of partial epilepsy at the Maudsley or King's College Hospitals, London between 1987 and 1999; $(b)$ patients were given a battery of noninvasive neuropsychological tests to estimate their cognitive functions (intelligence, verbal memory, and non-verbal memory) both pre- and post-surgically; (c) the laterality of speech and memory functions was assessed by the Wada test; and $(d)$ an angiogram preceding the Wada test did not show cross-filling to the non-injected hemisphere. Exclusion criteria were: (a) presence of clear neuroimaging abnormalities outside the suggested operated temporal lobe; and $(b)$ intelligence quotient below 70 .

The above criteria were met by 106 patients, of which 91 underwent TL (45 left and 46 right) and 15 had AH (9 left and 6 right). All patients were operated on by a single neurosurgeon (CEP) and the battery of neuropsychological tests, including the protocol for the Wada test, was set up and implemented by the same neuropsychologist (RGM). ${ }^{20}$ Postsurgical neuropsychological assessment was carried out 11 (13) months after the surgery (mean (SD)). All the patients signed an informed consent before the Wada test and before surgery.

Handedness was established either by Annett's Handedness Inventory ${ }^{21}$ or by non-structured questioning at the time of carrying out pre-surgical neuropsychological testing. 


\section{Wada test}

The protocol of the Wada test routinely performed in our centre as a pre-surgical procedure to assess memory and language lateralisation has been described in detail elsewhere. ${ }^{202-25}$ During the test, patients were monitored with scalp electroencephalographic recordings. Before intracarotid injections of sodium amobarbital to each hemisphere, standard internal carotid angiography was conducted to check that the distribution of contrast was unilateral and confined to the territories supplied by the anterior and middle cerebral arteries.

Left and right Wada tests were performed on the same day. Sodium amobarbital was injected at a rate of $25 \mathrm{mg} / 5 \mathrm{~s}$ until hemiparesis of the contralateral arm appeared. Usually the right sided injection test was carried out first, with the rationale of avoiding affecting the dominant hemisphere for language during the first run of the procedure. The mean (SD) dose of sodium amobarbital was 106.89 (22.54) $\mathrm{mg}$ for the left hemisphere injection and 108.32 (17.39) $\mathrm{mg}$ for the right. Immediately after injection, patients were shown 18 items mounted on $25 \times 12 \mathrm{~cm}$ file cards, one by one for 5 seconds each. Items consisted of a series of six words of medium or high frequency, ${ }^{26}$ six line drawings of common objects taken from a standardised set of pictures, ${ }^{27}$ and six white male faces printed in black and white, taken from the Spotlight catalogue of actors, mixed together in a pseudorandom order. Two parallel versions of the memory test were used, one for the left injection and another for the right. The presentation of the memory items took approximately 3 minutes. Language dominance was assessed clinically on the basis of observation of positive signs of dysphasia and an inability to read words and name the line drawings presented.

The procedure to measure recognition memory was performed 10 minutes after neurological examination, when scalp EEG had returned to baseline after injection. Eighteen target items were presented individually, mixed in a pseudorandom order with the same number of equivalent distracter items. Patients were instructed to respond "yes" if they had seen the item previously and "no" if not. If not certain, they were required to make a guess. After testing one hemisphere, the other hemisphere was tested following the same procedure.

For each hemisphere and each category (words, objects, and faces), true and false recognitions were counted. A score for each category and each hemisphere was calculated by subtracting the false from the true recognitions. In addition, for each hemisphere, a total memory score was calculated by adding all the scores in each category. Thus a total of 18 score marks for each test could be obtained if the patient correctly recognised all the items previously shown and rejected all the distracter items, and a score of -18 would be obtained if only distracters were chosen. A cut off level of 5 in the Wada score was chosen on the basis that in order to pass the test, a patient had to obtain a score unlikely to occur by chance if rendered amnesic $(\mathrm{p}<0.05)$. Memory laterality was considered as unilaterally low in patients obtaining scores $<5$ when injecting one side but not the other, bilaterally low in patients obtaining scores $<5$ when injecting on either side, and bilaterally high in patients obtaining scores $\geqslant 5$ when injecting on either side. The terms contralateral and ipsilateral refer to the operated hemisphere. A patient failed the Wada test if the total score obtained when testing the side contralateral to the proposed resection (that is, when injecting on the hemisphere ipsilateral to the resection) was $<5$. Likewise, ipsilateral Wada scores refer to the scores obtained when testing the operated hemisphere (when injecting the side contralateral to the operation), and contralateral Wada scores refer to the scores obtained when testing the hemisphere contralateral to the proposed operation (when injecting the side ipsilateral to the operation). Initially, 7 of the 13 patients who had TL and initially failed the Wada test underwent a second Wada test to rule out low Wada scores due to poor concentration or excessive dose of sodium amobarbital. Only one patient passed the Wada test on the second occasion and was removed from the group of patients who failed the test.

\section{Neuropsychological assessment}

All patients underwent neuropsychological evaluations as part of their pre- and post-surgical assessment. A battery of tests was designed to assess intellectual function, verbal memory, and visuospatial memory. The tests are used routinely in our and other centres for neuropsychological assessment in patients with epilepsy. ${ }^{28}$

Intellectual function was examined using a short form of the Wechsler Adult Intelligence Scale Revised, which includes the vocabulary, comprehension, similarities, block design, and object assembly sub-tests. ${ }^{29}$ This provides a prorated full scale intelligence quotient (FSIQ) as well as prorated measures of verbal and performance intelligence quotients (VIQ and PIQ).

The logical memory sub-tests of the Wechsler Memory Scale (WMS) were administered to assess verbal memory function. These sub-tests are used routinely for presurgical memory assessment in patients considered for temporal lobe resections. The immediate logical memory (WLMI) test includes two prose passages that are read to the patient and must be recalled immediately. A delayed version (WLMD) of this task was employed in which the patient was instructed to recall the passages after a delay of 1 hour.

Visuospatial memory was tested by using the delayed recall form of the Rey-Osterrieth figure (percentage recall), with an initial drawing of the figure and drawing from memory after 40 minutes; ${ }^{30}$ percentages of correct scores were computed.

To evaluate differences between pre- and post-surgical neuropsychological performances, we have calculated a change score for each neuropsychological test as:

$$
\text { change }=\left(\frac{\text { post }- \text { pre }}{\text { pre }}\right) \cdot 100
$$

where: change $=$ change score; pre $=$ pre-surgical score, and post $=$ post-surgical score.

This score estimates the percentage of change after surgery compared with preoperative levels. According to this formula, positive scores indicate an increase in the corresponding score after surgery, while negative scores indicate a decrease in the score after surgery. Thus, the higher the change scores, the better the neuropsychological performance after surgery.

Surgical techniques and selection criteria for TL or $\mathrm{AH}$ Anatomically standardised surgical techniques were used. ${ }^{31}$ En bloc temporal lobectomy was performed at the Maudsley and King's College Hospitals as originally described by Falconer. ${ }^{32}$ However, from 1991 the technique was modified to achieve a more complete removal of the hippocampus using the principles described by Spencer. ${ }^{33}$ In effect, between 5.5 and $6.5 \mathrm{~cm}$ of temporal lobe was removed. In the dominant hemisphere, usually the left, all superior temporal gyrus except the anterior $2 \mathrm{~cm}$ was spared. Such a resection would include at least $50 \%$ of the amygdala and $2-3 \mathrm{~cm}$ of parahippocampal gyrus and hippocampus. The extent of the resection was occasionally modified according to electrocorticographic findings.

AH has been performed at the Maudsley and King's College Hospitals between April 1987 and June 1995. Selective AH was performed using the technique described by Yasargil. ${ }^{5}$ This technique removes most of the parahippocampal gyrus, 
and the same amount of amygdala and hippocampus as the en bloc TL, leaving the majority of the lateral neocortex intact. $^{34}$

In general, TL was considered to induce more substantial memory deficits than $\mathrm{AH}$, as the latter removes far less temporal cortex. The specific criteria for offering TL were:

1. Evidence of temporal lobe epilepsy provided by a comprehensive battery of diagnostic tests (patient history, scalp interictal EEG, neuroimaging, PET, ictal scalp or intracranial EEG recordings); and

2. If one of the following two conditions occurred: $(a)$ the patient passed the Wada test, or $(b)$ the patient failed the Wada test and showed bilaterally low memory scores on the test in the presence of a clear unilateral focal structural abnormality in the temporal lobe proposed for resection, and presurgical neuropsychological tests showed normal scores.

It was felt that patients who fulfilled criterion $2 \mathrm{~b}$ appeared to show higher memory reserve than estimated by the Wada test, and therefore TL was justified. Twelve patients were offered TL after failing the Wada test on this basis. These 12 patients form a small but relevant sample, as they provide direct observation of the effects of TL on patients whose unresected hemisphere did not support memory according to the Wada test. However, it should be emphasised that these 12 patients represent a very selected population where we thought that TL was ethically justified despite failing the Wada test. The exclusion criterion for TL was to have failed the Wada test and to have failed to fulfil criterion $2 \mathrm{~b}$.

During the period of recruiting (1987-1999), inclusion criteria for $\mathrm{AH}$ varied slightly. In general, $\mathrm{AH}$ was carried out in patients in whom: (a) the epileptogenic focus was restricted to mesial temporal structures unilaterally on the basis of neuroimaging findings and/or of EEG telemetry with foramen ovale, subdural, or depth electrode intracranial implantations; and/or $(b)$ the patient failed the Wada test and criterion $2 \mathrm{~b}$ described above for TL was not fulfilled, suggesting that there was a risk of post-operative memory dysfunction from TL. ${ }^{35}$

From the above criteria, it can be seen that no patient was excluded from surgery on the basis of the Wada test, although the operation offered (TL or AH) depended on results from the Wada and whether the patient fulfilled criterion $2 \mathrm{~b}$ for TL.

Because patient selection for AH required stricter criteria regarding the topography and extension of seizure onset, and because patients passed the Wada test more frequently than failed it, the number of patients who underwent TL (91 patients) was necessarily larger than the number of patients submitted for $\mathrm{AH}$ (15 patients).

\section{Seizure control outcome}

The mean (SD) post-surgical follow up period was 42.8 (24) months. The number of seizures was recorded during the follow up period and the seizure outcomes were rated according to Engel's classification. ${ }^{16}$ A dichotomous grouping was then used, classifying outcome as favourable or poor. Favourable outcome included Engel class I (free of disabling seizures) and II (almost seizure free, having three or fewer seizures per year). Poor outcome included Engel class III (worthwhile improvement remaining with more than three seizures per year) and IV (no worthwhile improvement).

\section{Data analysis}

Data analysis was carried out with the Statistical Package for Social Sciences (version 10.0, 1999; SPSS Inc. Chicago, IL, USA). Nominal data were analysed by Yates' corrected $\chi^{2}$ test or Fisher's exact test. For comparison of patient groups, nonparametric statistics were preferred because of the relatively small size of some samples and non-normal distribution of some variables. Demographic characteristics and neuropsychological tests were compared between groups using the Wilcoxon test $(\mathrm{Z})$ for independent samples. Multiple comparisons in change scores among patient groups were also carried out using the Kruskal-Wallis test. Spearman's correlation coefficient $(\rho)$ was calculated to estimate the presence of a relationship between Wada scores and neuropsychological change scores. Differences were considered statistically significant if $\mathrm{p}<0.05$.

\section{RESULTS}

\section{Demographic characteristics}

The demographic characteristics of all the patients studied are summarised in table 1 . The patients who underwent AH showed a higher proportion of bilaterally low memory and a lower proportion of unilaterally low memory than those who underwent TL $(\mathrm{p}<0.05)$. As expected in accordance with the selection criteria described above, the proportion of patients who failed the Wada test when injecting the ipsilateral hemisphere was higher among patients who underwent $\mathrm{AH}$ than among those who had TL $(\mathrm{p}<0.01)$. Similarly, a higher proportion of mesial temporal sclerosis (MTS) among AH patients $(p=0.05)$ was to be expected, as evidence of a mesial temporal focus is an inclusion criterion for AH but not for TL. The groups of patients who underwent TL or AH did not show significant differences in the proportion of patients who enjoyed a favourable outcome with respect to seizure control (64\% versus $60 \%$ respectively). As failing the Wada test was an inclusion criterion for $\mathrm{AH}$, contralateral Wada scores were significantly lower among $\mathrm{AH}$ patients $(\mathrm{p}<0.001)$.

The demographic characteristics of the patients who failed the Wada test are also summarised in table 1. No differences

Table 2 Neuropsychological scores according to operation type

\begin{tabular}{|c|c|c|c|c|c|c|c|c|}
\hline \multirow[b]{3}{*}{ Test } & \multicolumn{4}{|c|}{ Pre-surgical scores } & \multicolumn{4}{|c|}{ Change scores } \\
\hline & \multicolumn{2}{|l|}{$\mathrm{TL}$} & \multicolumn{2}{|l|}{$\mathrm{AH}$} & \multicolumn{2}{|l|}{ TL } & \multicolumn{2}{|l|}{$\mathrm{AH}$} \\
\hline & $\mathbf{n}$ & Median (range) & $\mathbf{n}$ & Median (range) & $\mathbf{n}$ & Median (range) & $\mathbf{n}$ & Median (range) \\
\hline $\mathrm{FSIQ}$ & 91 & 91 (71 to 124) & 15 & 92 (83 to 108 ) & 91 & $1(-22$ to 28$)$ & 15 & $2(-23$ to 20$)$ \\
\hline VIQ & 91 & $88(74$ to 124$)$ & 15 & 91 (73 to 106) & 91 & $-1(-25$ to 25$)$ & 15 & $-2(-24$ to 19$)$ \\
\hline$P I Q$ & 91 & 96 (57 to 147$)$ & 15 & 92 (80 to 126 ) & 91 & $2(-19$ to 67$)$ & 15 & $0(-23$ to 28$)$ \\
\hline WLMI & 83 & 7.5 (1 to 17$)$ & 13 & 8 (3.5 to 14.5$)$ & 83 & $-14(-73$ to 363$)$ & 13 & $-14(-35$ to 113$)$ \\
\hline WLMD & 80 & $4.88(0$ to 13.5$)$ & 13 & $4.25(1.5$ to 12$)$ & 75 & $-13(-100$ to 1300$)$ & 12 & $28(-100$ to 206$)$ \\
\hline Rey $\%$ & 81 & 48.94 (0 to 91.49 ) & 13 & $52.17(27.66$ to 76.74$)$ & 79 & $5(-100$ to 435$)$ & 13 & $-9(-100$ to 115$)$ \\
\hline
\end{tabular}

$\mathrm{TL}$, temporal lobectomy; $\mathrm{AH}$, amygdalohippocampectomy; FSIQ, Full scale intelligence quotient; VIQ, verbal intelligence quotient; $\mathrm{PIQ}$, performance intelligence quotient; WLMI, Wechsler Memory Scale revised, logical memory, immediate recall; WLMD, Wechsler Memory Scale revised, logical memory, delayed recall; Rey \%, Rey figure, percentage recall of the copy. 
were found in these features between patients who underwent TL and AH.

\section{Comparison of the neuropsychological scores in patients who underwent TL or $\mathrm{AH}$}

Table 2 shows the neuropsychological scores (pre-surgery and change scores) for patients who underwent TL and those who had AH. No patient became globally amnesic after surgery. No differences in pre-surgical or change scores were found between both patient groups.

\section{Comparison of the neuropsychological scores according to operation type in patients who passed or failed the Wada test}

Table 3 shows pre-surgical neuropsychological scores and change scores in patient sub-populations according to operation type and to whether they passed or failed the Wada test.

Among the patients who passed the Wada test, change scores for Rey \% recall were higher (more positive) between patients who had TL than those who had AH $(\mathrm{p}<0.05)$. For the patients who failed the Wada test, there were no significant differences between patients who had TL and those who had AH; however, there was a strong trend in the WLMD for more positive (higher) change scores in patients who had AH than those who had TL $(\mathrm{p}=0.07)$.

Among the patients who underwent TL, there were no statistical differences in pre-surgical scores between patients who passed or failed the Wada test. Only the change score for Rey \% recall was significantly lower in the patients who failed the Wada test compared with those who passed $(\mathrm{p}<0.05)$.

Among the patients who underwent $\mathrm{AH}$, pre-surgical scores of the WLMI and WLMD measures were higher among patients who passed the Wada test than those who failed $(p<0.05, p<0.01$ respectively). Change scores of the WLMD were higher among those who failed the Wada test than among those who passed $(\mathrm{p}<0.05)$.

Comparison of change scores among the four patient groups using the Kruskal-Wallis test did not show significant differences.

In order to investigate the effects of laterality on change scores, the four patient groups (TL pass, TL fail, AH pass, AH fail) were further split according to operation side and to whether the operated side was ipsilateral or contralateral to language dominance. Unfortunately, most sample sizes became too small for statistical comparison, but no obvious differences appeared to exist between groups (see fig 1).

\section{Correlation between contralateral Wada scores and neuropsychological change scores}

As contralateral Wada scores estimate memory function of the hemisphere that remains after surgery, they are assumed to be a predictor of the effects of surgery on memory. We tested this hypothesis by studying the correlation between contralateral Wada scores and change scores for all neuropsychological tests used. Among all patients, there were low but significant positive correlations between contralateral Wada scores and FSIQ change scores $(p<0.05)$, and PIQ change scores $(p<0.05)$.

The 12 patients who had TL despite failing the Wada test are of particular interest in studying potential neuropsychological deficits after temporal resections, as these are the patients thought to be at risk of developing memory deficits. Interestingly, among these 12 patients, much higher and significant correlation coefficients were found, particularly for verbal function. In particular, there were significant positive correlations between contralateral Wada scores and VIQ change scores $(\mathrm{p}<0.01)$. In addition, there was also a

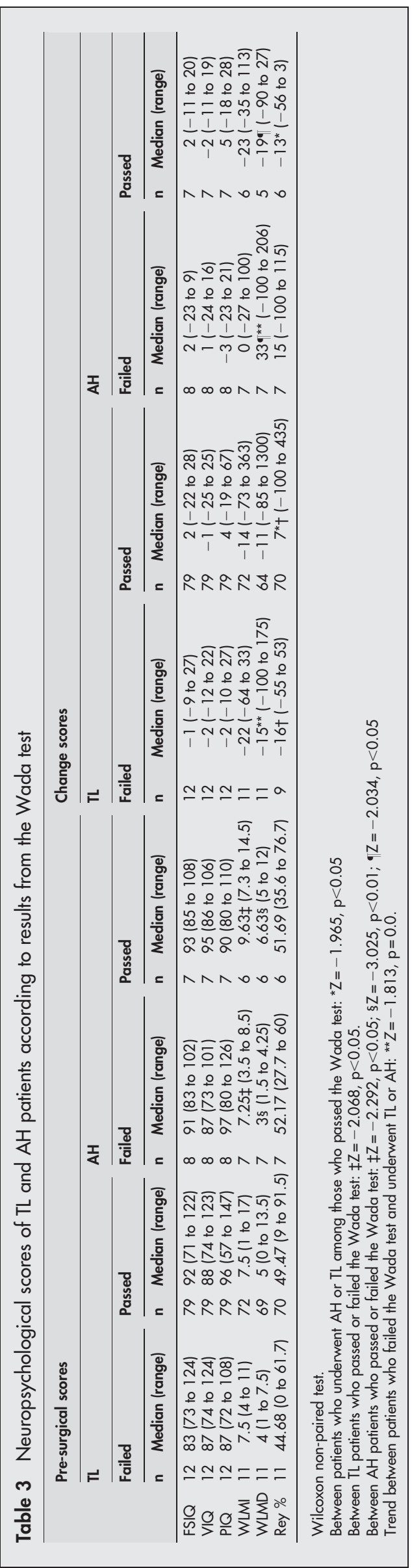



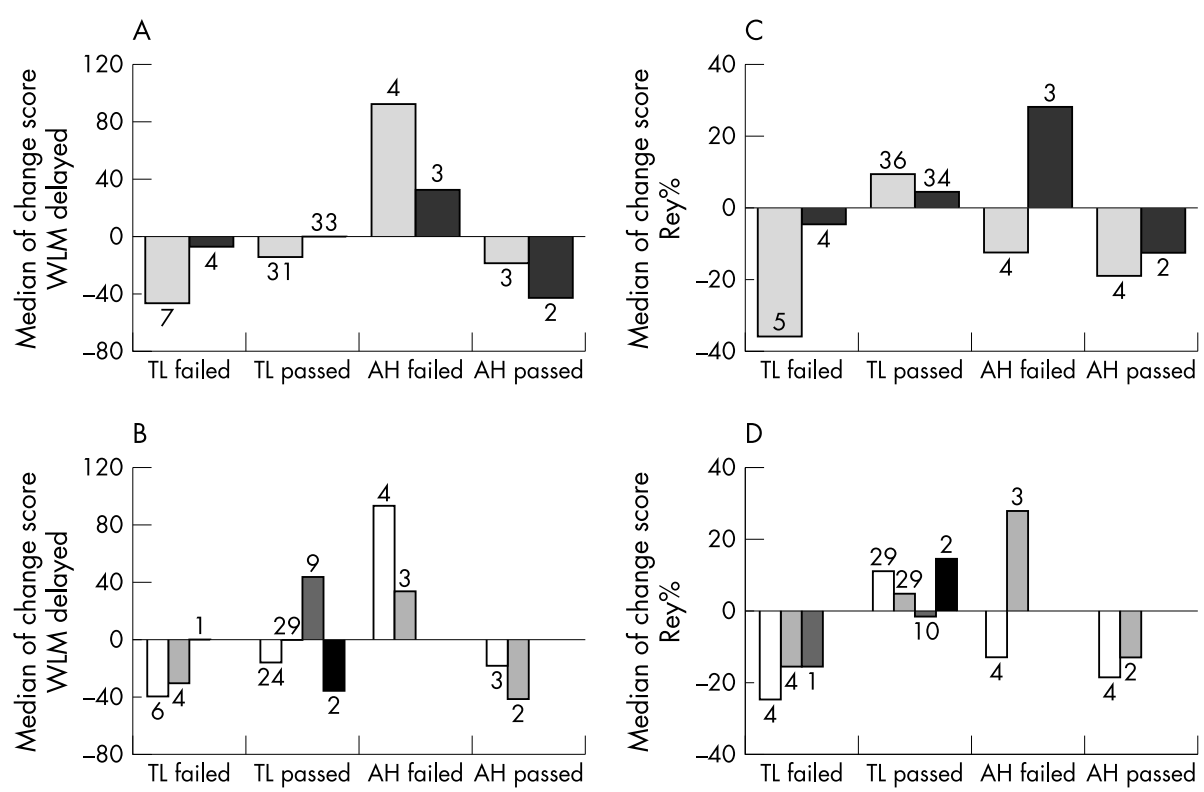

Figure 1 Medians of change scores in WLMD and Rey \% recall for patients who underwent $\mathrm{AH}$ or $\mathrm{TL}$ and passed or failed the Wada test. (A) Medians of change scores in WLMD according to the side of operation. (B) Medians of change scores in WLMD according to whether language dominance was ipsilateral or contralateral to the resection, bilateral, or not established. (C) Medians of change scores in Rey \% recall according to the side of operation. (D) Medians of change scores in Rey \% recall according to whether language dominance was ipsilateral or contralateral to the resection, bilateral, or not established. Numbers above or below each bar show the number of patients in each group.

Left $\square$ Ipsilateral $\square$ Contralateral

Bilateral language

Not established

Right

strong trend towards a correlation between contralateral Wada scores and change scores in WLMI $(p=0.06)$.

No significant correlations were found among the eight patients who had $\mathrm{AH}$ and failed the Wada test.

\section{DISCUSSION}

In general terms, we found few differences between the neuropsychological changes associated with TL or AH. No patient became amnesic after surgery, regardless of whether they had TL or AH, or whether they passed or failed the Wada test. No differences were found in pre-surgical or change scores of neuropsychological tests between patients who underwent TL or AH (table 2). These findings concur with those of Jones-Gotman et al, who showed no differences for verbal and non-verbal learning tasks after neocorticectomy, $\mathrm{TL}$, or $\mathrm{AH}^{14}$ Likewise, Wolf et al also found no major differences in cognitive performance with respect to the extent of left temporal resections. ${ }^{13}$ The findings are perhaps expected, as the Wada test is used preoperatively to tailor the operation in order to minimise the neuropsychological effects of temporal lobe resections.

We have extended the sample from Goldstein et al and found broadly similar results. In addition, in our present series, we included 12 patients who had TL despite failing the Wada test. These patients are of particular interest as they are considered to be at a higher risk of memory deficits after TL. Although we did not find gross differences between the neuropsychological effects of TL and $\mathrm{AH}$, patients who underwent TL and failed the Wada test showed more deficits than those who passed, or those who had AH. TL patients who failed the Wada test showed higher visual memory deficits after surgery than those who passed and, among patients who failed the Wada test, there was a strong trend suggesting a deficit in verbal memory following TL compared with patients who had AH. Indeed, deterioration in verbal memory among TL patients who failed the Wada test is confirmed by the presence of a correlation between contralateral Wada scores and verbal change scores, suggesting that those patients who show low contralateral Wada scores are at risk of verbal deficits after TL. Significant correlation coefficients were much higher among the 12 patients who failed the Wada test and underwent TL than within the general population or among those who had AH. This suggests that, as expected, the contralateral Wada scores are better predictors of neuropsychological outcome among those patients who fail the Wada and undergo a TL, as other patients are not expected to suffer significant neuropsychological deficits. Nevertheless, it is surprising that only relatively minor deficits were found in TL patients who failed the Wada test. In principle, this should be interpreted with caution because the number of patients who failed the Wada test is small and those who had TL after failing the Wada test were a highly selected patient population. However, deficits might be significantly higher among the general population of patients who fail the Wada test because our population of patients who had TL after failing is thought to include only patients with high memory reserve, and who were therefore at less risk of neuropsychological deficits after temporal resections. It cannot be ruled out that the absence of major differences in neuropsychological scores between TL and AH patients who failed the Wada test may be due in part to the effects of laterality of seizure onset and verbal dominance with respect to the resected side. Unfortunately, our samples were too small to investigate these issues thoroughly.

The neuropsychological tests administered were main outcome measures, but were limited in scope, so that these findings may be rather specific and may not be generalisable across other procedures. ${ }^{36}$ However, the logical memory test has been reported to be sensitive to the effects of temporal lobe lesions in several studies, ${ }^{9}{ }^{24}$ 37-39 showing similar deficits to those demonstrated by other memory tests such as the California Verbal Learning Test. ${ }^{36}{ }^{40}$ The sensitivity of the logical memory test to the degree of mesial temporal lobe resection has yielded variable results, with some authors reporting no difference ${ }^{41} 42$ whereas others showed significant differences. ${ }^{43}$

It may appear surprising that $\mathrm{AH}$ can be indicated in patients who fail the Wada test, as a relevant role for the hippocampus in memory has repeatedly been suggested. Studies in rats, ${ }^{44}$ monkeys, ${ }^{45}{ }^{46}$ and humans ${ }^{1}$ have shown that hippocampal lesions impair recognition memory. However, several studies in rats have not found major recognition memory impairment following hippocampal lesions, ${ }^{47}$ and it appears that in monkeys the hippocampus may not be as vital 
to memory encoding as the entorhinal or perirhinal cortices. ${ }^{48}$ Evidence from human studies also supports this view. In epileptic patients who underwent $\mathrm{TL}$, there seems to be no difference in material specific memory deficits regardless of whether the hippocampal resection was total or partial. ${ }^{43}$ Other authors have not found a relation between memory outcome and the extent of hippocampal/temporal resections. $^{8}{ }^{1213}$ In our study, the hippocampi removed from patients submitted to $\mathrm{AH}$ would be expected to be largely non-functional, as evidence of hippocampal epileptogenicity was an inclusion criterion for AH. Thus, it would not be surprising if in patients who underwent $\mathrm{AH}$ and failed the Wada test, extrahippocampal ipsilateral cortex was engaged in memory function. Alternatively it could be claimed that the absence of post-operative deficits in $\mathrm{AH}$ patients who fail the Wada test is due to the poor predictive value of the Wada test in AH patients, as the entire hippocampus is not irrigated by the internal carotid artery. ${ }^{49}$ Nevertheless, a functional effect is induced on the hippocampus during Wada testing, as EEG slowing in the posterior hippocampus has been shown following intracarotid amobarbital injection..$^{50}$

Among the patients who underwent $\mathrm{AH}$, those who passed the Wada test showed the highest relative deficits in WLMD, and patients who failed the test showed the highest improvements (table 3 ). The comparatively worse memory outcome in $\mathrm{AH}$ patients who passed the Wada test could be explained, at least in part, by the fact that these patients are coming from a higher preoperative baseline, and therefore have more to lose. ${ }^{36}$

In summary, we did not find profound changes in intelligence or memory scores after TL or AH. However, our findings suggest differences between TL and $\mathrm{AH}$, when patients had very low contralateral Wada scores. This is supported by a correlation between contralateral Wada scores and verbal change scores in patients who underwent TL after failing the Wada test, which was not found among patients who had AH after failing. Thus patients with particularly low contralateral Wada scores show a pronounced verbal function decline after TL but not after AH, suggesting that if surgery is required, $\mathrm{AH}$ might be preferred to TL in these patients.

\section{ACKNOWLEDGEMENTS}

This project was funded in part by the Fund for Epilepsy (London), the Japanese Epilepsy Research Foundation and Sección del Tercer Ciclo de la Universidad Autónoma (Madrid).

\section{Authors' affiliations \\ M E Lacruz, G Alarcón, N Akanuma, M Koutroumanidis, C D Binnie, C E Polkey, R G Morris, Division of Neuroscience, Guy's, King's and St. Thomas' School of Medicine, King's College Hospital, London, UK M E Lacruz, Departamento de Psicología Biológica y de la Salud, Universidad Autónoma, Madrid, Spain}

N Akanuma, Psychiatry and Behavioural Science, Tokyo Medical and Dental University Graduate School, Tokyo, Japan

F C K Lum, Department of Psychiatry, Chinese University of Hong Kong, Hong Kong, China

N Kissani, Department of Neurology, El Razi University Hospital, Marrakech, Morocco

N Adachi, Adachi Mental Clinic, Sapporo, Japan

Competing interests: none declared

\section{REFERENCES}

1 Scoville W, Milner B. Loss of recent memory after bilateral hippocampal lesions. J Neurol Neurosurg Psychiatry 1957;20:11-21.

2 Squire LR, Zola-Morgan S. The medial temporal lobe memory system. Science 1991;253:1380-6.

3 Eichenbaum $\mathbf{H}$. The hippocampus and declarative memory: cognitive mechanisms and neural codes. Behav Brain Res 2001;127:199-207.

4 Falconer MA, Serafetinides EA. A follow-up study of surgery in temporal lobe epilepsy. J Neurol Neurosurg Psychiatry 1963;26:154-65.
5 Wieser HG, Yasargil MG. Selective amygdalohippocampectomy as a surgical treatment of mesiobasal limbic epilepsy. Surgical Neurol 1982; 17:445-57

6 Wieser HG. Selective amygdalohippocampectomy: indications, investigative technique and results. Adv Tech Stand Neurosurg 1986;13:39-133.

7 Wieser HG. Selective amygdalohippocampectomy: indications and follow-up. Can J Neurol Sci 1991;18:617-27.

8 Goldstein LH, Polkey CE. Everyday memory after unilateral temporal lobectomy or amygdalo-hippocampectomy. Cortex 1992;28:189-201.

9 Goldstein LH, Polkey CE. Short-term cognitive changes after unilateral temporal lobectomy or unilateral amygdalo-hippocampectomy for the relief of temporal lobe epilepsy. J Neurol Neurosurg Psychiatry 1993;56:135-40.

10 Helmstaedter C, Elger CE, Hufnagel A, et al. Different effects of left anterior temporal lobectomy, selective amygdalohippocampectomy, and temporal cortical lesionectomy on verbal learning, memory, and recognition. J Epilepsy 1996:9:39-45.

11 Helmstaedter C, Grunwald T, Lehnertz K, et al. Differential involvement of left temporolateral and temporomesial structures in verbal declarative learning and memory: evidence from temporal lobe epilepsy. Brain Cogn 1997:35:110-31.

12 Pauli E, Pickel S, Schulemann H, et al. Neuropsychologic findings depending on the type of resection in temporal lobe epilepsy. Adv Neurol 1999;81:371-7.

13 Wolf RL, Ivnik RJ, Hirschorn KA, et al. Neurocognitive efficiency following left temporal lobectomy: standard versus limited resection. J Neurosurg 1993;79:76-83.

14 Jones-Gotman M, Zatorre RJ, Olivier A, et al. Learning and retention of words and designs following excision from medial or lateral temporal-lobe structures. Neuropsychologia 1997:35:963-73.

15 Polkey CE. Preoperative tailoring of temporal lobe resections. In: Engel J, ed. Surgical treatment of the epilepsies. New York: Raven Press, 1993:473-80.

16 Engel J, Van Ness PC, Rasmussen TB, et al. Outcome with respect to epileptic seizures. In: Engel J, ed. Surgical treatment of the epilepsies. New York: Raven Press, 1993:609-21.

17 Wada JA. A new method for determination of the side of cerebral speech dominance: a preliminary report on the intracarotid injection of sodium amytal in man. Igaku Seibutsugaku (Med Biol) 1949:4:221-2.

18 Wada JA, Rasmussen T. Intracarotid injection of sodium amytal for the lateralization of cerebral speech dominance: experimental and clinical observations. J Neurosurg 1960;17:266-82.

19 Milner B, Branch C, Rasmussen T. Study of short-term memory after intracarotid injection of sodium amytal. Trans Am Neurol Assoc 1962;87:224-6.

20 Morris RG, Polkey CE, Cox T. Independent recovery of memory and language functioning during the intracarotid sodium amytal test. J Clin Exp Neuropsychol 1998;20:433-44.

21 Annett M. The binomial distribution of right, mixed and left-handedness. QJ Exp Psychol 1967; 19:327-33

22 Morton N, Polkey CE, Cox T, et al. Episodic memory dysfunction during sodium amytal testing of epileptic patients in relation to posterior cerebral artery perfusion. J Clin Exp Neuropsychol 1996;18:24-37.

23 Ferrier $\mathrm{CH}$, Alarcon G, Glover A, et al. N-Acetylaspartate and creatine levels measured by (1)H MRS relate to recognition memory. Neurology 2000:55:1874-83.

24 Graydon FJ, Nunn JA, Polkey CE, et al. Neuropsychological outcome and the extent of resection in the unilateral temporal lobectomy. Epilepsy Behav 2001;2:140-51.

25 Akanuma N, Alarcon G, Lum F, et al. Lateralising value of neuropsychological protocols for presurgical assessment of temporal lobe epilepsy. Epilepsia 2003;44:408-18.

26 Francis WN, Kuzera $\mathrm{H}$. Frequency analysis of English usage: lexicon and grammar. Boston: Houghton Mifflin, 1982.

27 Snodgrass JG, Vanderwart M. A standardized set of 260 pictures: norms for name agreement, image agreement, familiarity, and visual complexity. J Exp Psychol (Hum Learn) 1980;6:174-215.

28 Rausch R, Le MT, Langfitt JT. Neuropsychological evaluation-adults. In: Engel J, Pedley TA, eds. Epilepsy: a comprehensive textbook. New York: Raven Press, 1997:977-88.

29 Canavan AG, Dunn G, McMillan TM. Principal components of the WAIS-R. B J Clinl Psychol 1986;2:81-5

30 Osterrieth PA. Le test de copie d'une figure complex. Arch Psychologie 1944;30:206-356.

31 Binnie CD, Polkey CE. Surgery for epilepsy. In: Matthews WB, ed. Recent advances in clinical neurology. Edinburgh, New York: Churchill Livingstone, 1992:55-93.

32 Falconer MA. Genetic and related aetiological factors in temporal lobe epilepsy. A review. Epilepsia 1971;12:13-31.

33 Spencer DD, Spencer SS, Mattson RH, et al. Access to the posterior medial temporal lobe structures in the surgical treatment of temporal lobe epilepsy. Neurosurgery 1984;15:667-71.

34 Renowden SA, Matkovic Z, Adams CB, et al. Selective amygdalohippocampectomy for hippocampal sclerosis: postoperative MR appearance. Am J Neuroradiol 1995;16:1855-61.

35 Olivier A. Commentary: cortical resections. In: Engel J, ed. Surgical treatment of the epilepsies. New York: Raven Press, 1987:405-16.

36 Hermann BP, Seidenberg M, Haltiner A, et al. Relationship of age at onset, chronologic age, and adequacy of preoperative performance to verbal memory change after anterior temporal lobectomy. Epilepsia 1995; $36: 137-45$. 
37 Hermann BP, Wyler AR. Neuropsychological outcomes of anterior temporal lobectomy. J'Neurosurg 1988;71:327-34.

38 Phillips NA, McGlone J. Grouped data do not tell the whole story: individual analysis of cognitive change after temporal lobectomy. J Clin Exp Neuropsychol 1995;17:713-24.

39 Smith ML. Memory disorders associated with temporal-lobe lesions. In: Boller F, Grafman J, eds. Handbook of neuropsychology. Amsterdam: Elsevier Science Publishers, 1989:91-106.

40 Martin RC, Sawrie SM, Roth DL, et al. Individual memory change after anterior temporal lobectomy: a base rate analysis using regression-based outcome methodology. Epilepsia 1998;39: 1075-82.

41 Milner B. Brain mechanisms suggested by studies of temporal lobes. In: Darley FL, ed. Brain mechanisms underlying speech and language. New York: Grune \& Stratton, 1967:122-45.

42 Ojemann GA, Dodrill CB. Verbal memory deficits after left temporal lobectomy for epilepsy. J Neurosurg 1985;62:101-7.

43 Wyler AR, Hermann BP, Somes G. Extent of medial temporal resection on outcome from anterior temporal lobectomy: a randomized prospective study. Neurosurgery 1995;37:982-90.
44 Clark RE, West AN, Zola SM, et al. Rats with lesions of the hippocampus are impaired on the delayed nonmatching-to-sample task. Hippocampus $2001 ; 11: 176-86$.

45 Alvarez P, Zola-Morgan S, Squire LR. Damage limited to the hippocampal region produces long-lasting memory impairment in monkeys. J Neurosci 1995; 15:3796-807.

46 Zola SM, Squire LR, Teng E, et al. Impaired recognition memory in monkeys after damage limited to the hippocampal region. J Neurosci 2000;20:451-63.

47 Mumby DG. Perspectives on object-recognition memory following hippocampal damage: lessons from studies in rats. Behav Brain Res 2001;127:159-81.

48 Murray EA, Mishkin M. Object recognition and location memory in monkeys with excitotoxic lesions of the amygdala and hippocampus. J Neurosci 1998; 18:6568-82.

49 Jack CR, Nichols DA, Sharbrough FW, et al. Selective posterior cerebral artery amytal test for evaluating memory function before surgery for temporal lobe seizure. Radiology 1988;168:787-93.

50 Gotman J, Bouwer MS, Jones-Gotman M. Intracranial EEG study of brain structures affected by internal carotid injection of amobarbital. Neurology 1992;42:2136-43. 